\title{
The Common Topological Origin of Waves in Quantum and Photon Physics as Well as in the Cosmic Redshift
}

\section{Ernst Karl Kunst}

Im Spicher Garten 5, 53639 Königswinter, Germany

Correspondence to: Ernst Karl Kunst, ernstkunst@aol.com

Keywords: Waves in Particle and Photon Physics, Cosmic-Redshift, Wavelike Pattern, Topological Interpretation, Cause of Mass and Time

Received: December 31, $2021 \quad$ Accepted: February 25, $2022 \quad$ Published: February 28, 2022

Copyright (c) 2022 by author(s) and Scientific Research Publishing Inc.

This work is licensed under the Creative Commons Attribution International License (CC BY 4.0).

http://creativecommons.org/licenses/by/4.0/

\section{(c) (i) Open Access}

\section{ABSTRACT}

It is shown that the well-known wave behaviors of material particles and photons, as well as the newly discovered wave-like structures in the cosmic redshift, are related phenomena that follow conclusively when senders and receivers of photons or material particles are topologically located in manifolds with a dimension difference of one. In this context, the inertial mass of the proton and the electron, their spin properties and the cause of time are derived from basic topological and physical laws. In addition, the quantum geometric basis of relativistic time dilation, the basis of the relativistic energy-momentum relationship and the relationship between energy and time are shown. Finally, it is shown that a curved cosmic space causes a distance-dependent reddening of light and the associated apparent escape velocity of distant cosmic objects, and that this also leads to a topologically conditioned wave structure of this redshift.

\section{INTRODUCTION}

It is generally known that the currently prevailing wave paradigm of material particles was introduced by Louis de Broglie through applying the Einsteinian energy equation of photons $E_{p h o t}=h v$ or $\lambda_{p h o t}=$ $h c / E_{p h o t}=h / p_{p h o t}$ onto single material particles with the non-relativistic result $\lambda_{B}=h p^{-1}[1,2]$. From this very origin of the particle-wave concept, the further path to current quantum theory mainly has been paved by Werner Heisenberg, Erwin Schrödinger and Paul Dirac [3-5]. But of course, the outstanding contributions to interpreting the strange-looking conclusions of the emerging quantum theory, in particular by Albert Einstein, Niels Bohr, Max Born, Pascual Jordan, John S. Bell and David Bohm, to name just the best-known physicists and natural philosophers, are no less important [6-11]. Currently, it is not doubted that the many equation systems for various quantum physical phenomena, based on the Schrödinger equation, with vectors in complex Hilbert spaces as their basic mathematical elements, correctly map the quantum physical reality. That means, quantum mechanics is expressed in phase space and 
not in space-time, whereas in contrast quantum mechanics in space-time is described through the path integral formalism of Dirac and Feynman.

On the other hand, the discussion about the correct interpretation of the theory, i.e. of the wave-function, is still going on. Out of the fullness of interpretation proposals the best known are:

1) The Kopenhagen interpretation introduced through Bohr and Heisenberg, mainly resulting from intense discussions of the former and Einstein about the correct interpretation of the theory, which is still considered the standard interpretation. In short: The physical world is unknowable and indeterminate such that "... the role of quantum-mechanics immediately is reduced to the generation of formal rules for the computation of the probabilities of the different observational results," (Fock, 1952) [12]. Known as the concept of complementarity, according to which a quantum object is both particle and wave, but never at the same time. Einstein never accepted the self-consistency of Bohr's argumentation.

2) The interpretation of the quantum theory through hidden variables by de Broglie (1927) and Bohm (1952). The authors interpret the wave-function of an individual particle as the mathematical expression of an objectively real field, such that the latter acts as a real force, analogously to the physical action of the electromagnetic or the gravitational field. Thus, no deviations from the wave-function can occur, but the cause of that field remains as elusive as the quantum physical phenomena, it claims to resolve.

3) The ambitious interpretation by Hugh Everett (1957), widely known as the "many-world interpretation" [13]. The Schrödinger equation is supposed to exactly reflect quantum-mechanical reality, implying that all probability-solutions expressed through the wave-function become reality physically in other parallel worlds.

It seems that currently none of the known proposals is accepted as really convincing. The question, whether quantum mechanics is complete, still remains unanswered, i.e., does in the end a physical-rationally founded ultimate cause of the wave properties of material particles and photons exist? Dirac's (similar: Feynman, but also Bohr and followers, see above) point of view, that quantum mechanics is complete, since the functions predict experimental results with sufficient accuracy and hence it seems senseless to ask for a deeper rooting physical basis, cannot be accepted. The latter position definitely corresponds to a pre-Copernican, Ptolemaic, non-physical way of thinking [14]. It is just not enough to constantly "improve" the laws of quantum physics, similar to the false concept of the earth at the center of the universe in the Middle Ages, through ever new, purely geometric-mathematical additional assumptions, but to formulate them in such a way that the physical reasons for the observed effects are listed.

\section{GENERAL PHYSICAL CONDITIONS FOR A TOPOLOGICAL INTERPRETATION OF WAVE STRUCTURES IN QUANTUM PHYSICS AND THE COSMIC REDSHIFT}

It should be noticed, that current theory tacitly assumes emission and reception of photons and material particles and their propagation physically to happen in real three-dimensional space with one additional time-like dimension (space-time), although the wave-functions are defined on an abstract, mathematical configuration space. Thus, topologically, the physical procedure of emission and reception of any particle, as well as its propagation in real space, also can be understood as bijective mapping $V_{3} \in \mathrm{R}^{3} \rightarrow V_{3}^{\prime} \in \mathrm{R}^{3}$, which is absolutely continuous, i.e. homeomorphic. Of course, in real space-time the translational motion of material particles as the carriers of the mapping, other than pure topological mapping, must obey special relativistic kinematics. Obviously does this notion of trajectories in three-dimensional space, including its relativistic extension as topological mapping in $\mathrm{R}^{3}$, indeed match classical physics but not the wavelike pattern of quantum physics and optics, which is continuous but under no circumstances bijective. Obviously is the deviation of the latter property of quantum physics and optics, from expectations on the grounds of the classical notion of the physical behavior of matter and light, the decisive point, about which all attempts for an explanation, beginning with Newton's theory on the nature of light, now for nearly four centuries circulate, without any satisfactory result so far.

But notice, that the above topological properties can be fulfilled through $V_{4} \in \mathrm{R}^{4} \rightarrow V_{3} \in \mathrm{R}^{3}$, i.e. topological mapping of the physical procedure of emission of whatever particle from four-dimensionality 
spatially with reception in three-dimensional space-time, as will be shown more detailed below.

Furthermore, it has been proposed the Universe to be a closed, static, self-containing Einsteinian three-sphere $\mathrm{S}^{3}$, spinning steadily in four-dimensional space spatially, with Euclidian $\mathrm{E}^{4}$ metric geometrically (locally), and neither expanding nor retracting [15]. As one consequence, among others, results "gravitation" not to be an "attractive central force", neither in the Newtonian sense nor in the sense of the Einsteinian space-force of general relativity, but rather a sheer apparent or pseudo force in form of the cosmic centrifugal force $\left[\omega_{P} P\right]^{2}=c^{2}$, which presses into and inside every Schwarzschild field towards the central mass.

The proton's mass has been derived from the impact of the Universe's centrifugal force onto the former's microscopic twofold four-dimensional height in dependence on the latter's cosmic latitude (see Chapter 3.1; electron 3.2). Whereas the Hubble (cosmological) redshift in this cosmological notion is due to the four-dimensional orthogonal displacement spatially of the emitter from the receiver's tangential pseudo Euclidian Minkowski-space in the three-sphere $S^{3}$, and not to a cosmological expansion (see Chapter 5.1.). In the following will be shown, that the latter propositions in association with the mentioned topological findings fully seem to explain the wave-like behavior of light and matter in optics and quantum physics as well as the observed wavelike pattern in the redshift of cosmological objects.

\section{PROPOSED TOPOGRAPHY OF MICRO-PHYSICS}

\subsection{Four-Dimensional Topography of the Proton on Quantum Scale and the Physical Origin of \\ Time}

According to the ref. No. [15] the mass of the only other stable fermion in free space besides the electron, the proton (antiproton), results from the rotation of the $S^{3}$ sphere (assumed to be at rest in $\mathrm{R}^{4}$ ), so that within the sphere the centrifugal energy

$$
E_{P}=\frac{m_{0}}{2}\left[\omega_{P} P\right]^{2}=\frac{1}{2} m_{0} c^{2}
$$

is generated. In association with the de Broglie-formula delivers the former equation the four-dimensional height $\lambda_{4} \equiv \lambda_{w}=\sqrt{h}=0.814013 \times 10^{-13} \mathrm{~cm}$ such that reversely applies

$$
m_{0}=\frac{2 \lambda_{w}(1-\sin A)}{c} \equiv \frac{2 \sqrt{h}(1-\sin A)}{c}=2 \tau_{w}(1-\sin A)
$$

angle $A$ (capital ALPHA) $=43.788^{\circ}$ being the Galaxy's latitude in the "northern hemisphere" (arbitrary definition) of the spinning Universe, $h$ Planck's constant, $m_{0}$ rest mass of proton, $c_{A}$ speed of light at cosmic latitude $A$ and $\lambda_{w} / c \equiv \lambda_{4} / c=\tau_{w}=\sqrt{h} / c$ quantum of (rest) time (see Chapter 5.). "This implies, contrary to common view, all fermionic material spatially to be build up four-dimensionally, although with the extremely tiny fourth spatial dimension $2 \lambda_{w}$ ". If the preceding is true, then the instability of all fermion masses (resonances) $>m_{0(A)}$ (proton) $=2 \tau_{W(A)}$, of the meson masses $<m_{0(A)}$ and of the free neutron must be due to the fact that their four-dimensional height $\neq 2 \lambda_{w}$. This requires the mass of the only other stable particle in free space, the electron (positron), for reasons of elementary topology to be a fixed stable fractional amount of $m_{0}$ (see Chapter 3.2). In accord with the preceding must the Euclidian quantum of time be of value

$$
\tau_{w}=\frac{\sqrt{h}}{c}=2.715256 \times 10^{-24} \mathrm{~s}
$$

Equations (2) and (4) are in accord with the CGS-system such that especially is valid (referred to $\mathrm{R}^{4}$ ): $m(\mathrm{~g})=\lambda_{W}(\mathrm{~cm}) / c=\tau_{W}(\mathrm{~s})$ and $\sqrt{h}$ really results in $\lambda_{W}(\mathrm{~cm})$ [15].

Now, how does $\tau_{w}$, or duration on quantum scale, come into existence physically? I.e., if we demand the cosmos really to be built-up as an Einsteinian hypersphere (hyper-ellipsoid) and material particles to exist as smallest possible geometrically four-dimensional objects rooting in the geometrically three-dimensional 
"surface" of the hypersphere. In the following is proposed that rest time principally is representable as the duration of the physical mapping of four-dimensional $\lambda_{W}$ into the geometrically three-dimensional rest frame of the respective particle $\lambda_{w} \rightarrow \tau_{w} c$. This also can be written as the difference

$$
\begin{gathered}
-\lambda_{x}^{2}+\lambda_{y}^{2}+\lambda_{z}^{2}-\lambda_{w}^{2}=0 \\
-\lambda_{x}^{2}+\lambda_{y}^{2}+\lambda_{z}^{2}-\tau_{w}^{2} c^{2}=0 \\
\lambda_{w}=\tau_{w} c
\end{gathered}
$$

because for four-dimensional $\lambda_{W}$ no equivalent exists in the geometrically three-dimensional space of our world, whereby $\lambda_{W}$ and $\lambda_{x}$ belong together in a special way, as we shall show immediately.

Other as the three spatial dimensions of space-time is $\lambda_{w}$ linked to the latter dimensions only in the dimensionless tie point $-\lambda_{x}^{2}+\lambda_{y}^{2}+\lambda_{z}^{2}-\lambda_{w}^{2}=0$. This implies that the rigid four-dimensional quantum of length can be mapped into $\mathrm{R}^{3}$ at the latter tie point firstly as the quantic continuum $\tau_{w}$ of points $\mathrm{d} \tau_{W}$ with highest possible velocity $\omega_{P} \equiv c=$ const. such that be valid $\mathrm{d} \lambda_{w}=\omega_{P} \mathrm{~d} \tau_{w}$ and thus:

$$
\tau_{w}=\frac{1}{\omega_{P}} \int_{0}^{\lambda_{w}} \mathrm{~d} \lambda_{w}=\frac{1}{C} \int_{0}^{\lambda_{w}} \mathrm{~d} \lambda_{w}=\int_{0}^{\tau_{w}} \mathrm{~d} \tau_{w}
$$

Secondly this physical-topological notion of the quantum of time evidently is associated with the proton's four-dimensional height $2 \lambda_{W}$ and the associated inertial mass according to Equation (2) in the form

$$
m_{0}=2 \int_{0}^{\tau_{w}} \mathrm{~d} \tau_{w}=2 \tau_{w}
$$

Thirdly, the four-dimensional $2 \lambda_{w}$ is considered to rotate in four-dimensional space around the tie point 0 (zero) perpendicular to its three-dimensional embedding, with the zero point between the two $\lambda_{w}$. This four-dimensional rotation of the fermions is perceived as spin of their hexagonal diameter in physical measurements (see Chapter 3.3 below).

The presented quantum-geometric notion of the origin of time as well as the protonic mass also leads directly to the physical basis of the relativistic time dilation and the energy-momentum relation. To this end, abstract from the dimensions $\lambda_{y}$ and $\lambda_{z}$ so that the tie point 0 (zero) is formed by the mutually perpendicular dimensions $\lambda_{x}$ and $\lambda_{w}$. Consider the four-dimensional $\lambda_{W}$ and thus the tie point now moving along the three-dimensional axis $2 \lambda_{x}$ with velocity $v$. The direction of $\lambda_{x}$ is given by the velocity vector $v$ such is valid: $v 2 \tau_{x}^{\prime 2}=v \lambda_{w}^{\prime} / c=v \tau_{w}^{\prime}$ until the tie point meets the movement of $\omega_{P}=c$ along $\lambda_{w}$ which becomes relative to the moving tie point to $c \tau_{w}^{\prime}=\lambda_{w}^{\prime}$. With this we have a right-angled triangle with the catheters $c \tau_{w}$ and $v \tau_{w}^{\prime}$ and the hypothenuse $c \tau_{w}^{\prime}$, so that the Pythagorean expression holds:

$$
\begin{gathered}
c^{2} \tau_{w}^{\prime 2}-\tau_{w}^{\prime 2} v^{2}=c^{2} \tau_{w}^{2} \\
\tau_{w}^{\prime 2}\left(c^{2}-v^{2}\right)=c^{2} \tau_{w}^{2} \\
\tau_{w}^{\prime}=\frac{\tau_{w}}{\sqrt{1-\frac{v^{2}}{c^{2}}}}
\end{gathered}
$$

From Equation (7) the relativistic energy-momentum relation of the proton, $E^{\prime 2}-E^{\prime 2} \beta_{0}^{2}=E^{2}$ directly follows through the demand that $2 \lambda_{W}$ is always conserved and, thus, by multiplying both sides of the second line of Equation (7) by $4 c^{2}$ follows directly:

$$
\frac{4 \lambda_{w}^{2}\left(c^{2}-v^{2}\right)}{1-\frac{v^{2}}{c^{2}}}=4 \lambda_{w}^{2} c^{2}=m_{0}^{2} c^{4}=E_{0}^{2}
$$


Factor $(1-\sin A)$ is neglected. I.e., the fundamental conservation of four-dimensional elementary length $\lambda_{w}=m_{0} c /[2(1-\sin A)]=E_{0} /[2 c(1-\sin A)]$ in the special relativistic energy conservation law (first left-hand sided ratio of Equation (8)) seems to be the very origin of the association of rest energy $E_{0}$ and rest mass $\mathrm{m}_{0}$ of the proton but of the electron as well and, therewith, of all stable matter. This demand is justified, since beyond the tie point time does not exist, so that the four-dimensional centrifugal energy of $c \tau_{W}+\lambda_{W}=2 \lambda_{W}$ of (1) is mapped to the quantum geometric environment (metric) of the tie point according to the Einstein general relativity theory as a deviation from the undisturbed flat Euclidian metric $\eta_{n m}$ :

$$
\varkappa T_{m n}=\frac{8 \pi G}{c^{2}} T_{m n}=2 U=2 E_{0}=2 \tau_{w}\left[\omega_{P} P\right]^{2}=2 \lambda_{w} c=m_{0} c^{2}=R_{m n}-\frac{1}{2} R g_{m n}
$$

The energy-momentum tensor $T_{m n}$ (cosmic latitude A neglected) represents the distribution of protonic matter (energy), and the Ricci tensor $R_{m n}$ the respective symmetric warping of space. In Newtonian approximation, constant $\varkappa$ of the left-hand side of the Einsteinian field equation can be replaced through the respective value $\varkappa=8 \pi G / \mathcal{c}^{2}, T_{m n}$ through $T_{44}=\varrho c^{2}$. If now the Poisson potential $U=4 \pi G \varrho$ of classical gravity is introduced, then eventually $\varkappa T_{m n}=2 U$ follows. If we replace the Poisson potential $U$ by the centrifugal energy $E_{0}$ of the resting proton according to Equation (1), then the left-hand side of the Einsteinian field equation takes the form of Equation (9). Thus in the result, firstly the quantum geometric basis of the relativistic time dilation and secondly of the relativistic energy-momentum relation is revealed, third the already longer assumed connection between energy and time.

One also should notice the numerical value $\lambda_{W}=0.814013 \times 10^{-13} \mathrm{~cm}$ of the proton's four-dimensional geometric height to be very near to the recent experimental finding that the former's charge radius $\approx$ $0.8335(95) \times 10^{-13} \mathrm{~cm}$. It is clear that the apparent rest time continuum of any observation every observer in the three-sphere will experience in reality is the finite sum

$$
\sum_{0}^{\Delta t} \tau_{w}=\Delta t
$$

of the finite time quanta $\tau_{w}=\sqrt{h} / c$ associated with $\lambda_{w}$ and that the physical presence really lasts only $\tau_{w}$ second with reference to the respective rest-frame in the sphere. This explains why also in quantum physics time always takes a "sharp" (Feynman) value. It is clear that according to the preceding this must be valid for mass and energy as well. Furthermore, a speculative time travel is not possible since existing time quanta can be relativistic delayed though, but, other than assumed by Gödel, past and future time quanta have no physical existence.

\subsection{Proposed Four-Dimensional Topography of the Electron (Positron)}

Above has been shown that the rest mass of the only stable fermion next to the electron (positron) in the Universe, the proton (antiproton), is directly related to the four-dimensional time quantum $\tau_{w}=\lambda_{w} / c=\sqrt{h} / c$ in dependence on the degree of latitude in the order of magnitude $(1-\sin A)$. Because of this very basic nature of the proton's mass it is obvious, that the only other stable fermion with defined rest mass, the electron (positron) should be derivable from the former basically. As shown above, follows the relativistic association of the proton's mass and energy from the preservation of the four-dimensional height $\lambda_{W}$ of a double four-simplex. Now, it is suggested that the electron be build up on the same elementary four-dimensional scale as the proton, but with the variation that the basic three-dimensional quantum volumes of these basic particles, proton and electron (and their antiparticles), are not equal, such that one can write

$$
m_{e}=x \times 2 \tau_{w}(1-\sin A) \neq m_{p}
$$

where $x$ stands for the still to be found reduction of the three-dimensional volume fraction of $m_{p}$, which obviously has the value $\lambda_{1} \times \lambda_{2} \times \lambda_{3}=1$, to the smallest volume possible in nature. From the preceding it is 
clear that on this elementary scale between the individual Euclidic grid points $\lambda_{1}-\lambda_{3}$ other points do not exist so that on this smallest possible spatial scale a circle $2 \lambda_{1} \pi$ cannot be constructed within the microscopic $\lambda_{1}$-grid system. The same is true for the construction of the area of an elementary circle $\pi\left(\lambda_{2}\right)^{2}$ within the $\lambda_{2}$-grid and of a sphere $4 \pi\left(\lambda_{3}\right)^{2}$ within the $\lambda_{3}$-grid, respectively, with the consequence that radius $r_{e}$ must be other than the former quantum lengths.

Because, thus, in neither spatial dimension on quantum scale radius $r_{e}$ can be constructed from rational $\lambda_{1}$ nor irrational $\lambda_{2}$ and $\lambda_{3}$, respectively, this implies the former to be transcendental and of the order of magnitude of $1 / \pi$. I.e. besides the dimension-dependent quantum lengths $\lambda_{1}-\lambda_{3}$ a transcendental grid with $\pi$ in the denominator must exist to render construction of circular bodies on quantum scale in all three spatial dimensions. It is clear that within the $\pi$-grid, higher $\pi$-values can only be generated by multiplying once or more by $\pi$ itself. So the quantum $\pi$-units have to be derived for each spatial dimension individually, depending on the respective smallest length of the three spatial dimensions:

$$
\frac{\lambda_{1}^{2}}{\pi_{1}^{1}} \Rightarrow \sqrt{\frac{\lambda_{1}^{2}}{\pi^{1}}}=\frac{\lambda_{1}}{\sqrt{\pi}}, \frac{\lambda_{2}^{2}}{\pi_{1}^{1} \cdot \pi_{2}^{2}} \Rightarrow \sqrt{\frac{\lambda_{2}^{2}}{\pi^{3}}}=\frac{\lambda_{2}}{\pi \sqrt{\pi}}, \frac{\lambda_{3}^{2}}{\pi_{1}^{1} \cdot \pi_{2}^{2} \cdot \pi_{3}^{3}} \Rightarrow \sqrt{\frac{\lambda_{3}^{2}}{\pi^{6}}}=\frac{\lambda_{3}}{\pi^{3}} .
$$

Of course, be valid $\pi_{1} \equiv \pi_{2} \equiv \pi_{3}$. Thus, the mass ratio $\mathrm{m}_{\mathrm{p}} / \mathrm{m}_{\mathrm{e}}$ takes the form

$$
\frac{m_{p}}{m_{e}}=2 \tau_{w}(1-\sin A) \lambda_{1} \lambda_{2} \lambda_{3} \cdot\left(2 \tau_{w}(1-\sin A) \frac{\lambda_{1} \lambda_{2} \lambda_{3}}{3 ! \cdot \sqrt{\pi} \cdot \pi \sqrt{\pi} \cdot \pi^{3}}\right)^{-1}=6 \pi^{5}
$$

where 3 ! in the denominator results from the multiplication of $\lambda_{1}, \lambda_{2} / 2$ and $\lambda_{3} / 3$ to receive in connection with Equation (11) the smallest possible volume of the electron. Therefore is given:

$$
\frac{2 \tau_{w}(1-\sin A)}{6 \pi^{5}} \equiv \frac{m_{p}}{6 \pi^{5}}=9.10956 \times 10^{-28} \mathrm{~g}, \frac{m_{p}}{m_{e}}=1836.118109
$$

One should notice the latter value to be not quite, though very near to the tabular one $m_{e}=$ $9.109387015 \times 10^{-28}$ and $m_{p} / m_{e}=1836.152668$. The reason is that both, $m_{p}$ and $m_{e}$ are considered as masses resting in their respective rest frame. However, this does not apply to the electron, because its mass is so tiny that it can only be measured in the orbit about a resting atomic nucleus with known rest mass. But according to the symmetric extension of special relativity, every earthly observer resting in her or his frame of reference considers her or his rest frame to be resting relative to the CMB, whereas the respective atom, consists of the nucleus at rest and the orbiting electron. Therewith the latter translationally is moving with $V_{0}(\mathrm{CMB}) \approx 370 \mathrm{~km} \cdot \mathrm{s}^{-1}$ relative to the $\mathrm{CMB}$. It is thus to be expected that for every observer, who is at rest relative to the $\mathrm{CMB}$, the volume and therewith the mass of the respective electron as well as its orbit will appear relativistically stretched due to this one-way propagation of the atom relative to the CMB by the amount of

$$
\Delta x_{(1-\text { way })}=370 \times \Delta T_{(1-\text { way })}=370 \times \frac{\Delta x_{01(v \top) 1 \text {-way }}-\Delta x_{01(1-\text { way })}}{2 \times \pi \times 370 \times \Delta t_{01}}
$$

With unit 1 (one) for one electron's mass this takes the form:

$$
\Delta m_{e(1-\text { way })}=\left[\left(1-\frac{370^{2}}{c^{2}}\right)^{-1}-1\right] \times \frac{370^{2}}{2 \times \pi} \times 1=0.03318362127
$$

In view of this result, Equation (14) becomes to

$$
\frac{m_{p}}{6 \pi^{5}}=9.10956 \times 10^{-28} \mathrm{~g}, \frac{m_{p}}{m_{e}}=1836.151293
$$

Notice that the latter value comes very near to the experimental one $m_{p} / m_{e}=1836.152668$. The slight deviation is most probably due to a slightly higher speed relative to the CMB than the above accepted 370 
$\mathrm{km} / \mathrm{s}$. E.g. calculation with $V_{\mathrm{CMB}}=373 \mathrm{~km} / \mathrm{s}$ results in $m_{p} / m_{e}=1836.152386$ and with $V_{\mathrm{CMB}}=374 \mathrm{~km} / \mathrm{s}$ in 1836.152749. Finally, it should be mentioned that Lenz pointed out in a short article as early as 1951 that the ratio $m_{p} / m_{e}$ comes very close to the product $6 \pi^{5}[16]$.

\subsection{Proposed Four-Dimensional Topography of the Fermions Explains the Spin and More}

The proposed four-dimensionality of the fermions proton (antiproton) and electron (positron) also explains two strange properties that contradict the view and the known physics. On the one hand, this is the experimental fact that a rotation of the axis of rotation by 360 degrees does not correspond to the initial value, e.g. + (plus), but - (minus) results and only after a further rotation by 360 degrees results again + (plus). I.e. two full rotations of 360 degrees each are required to reach the initial value again, while in the geometrically three-dimensional world of our view a rotation of 360 degrees is sufficient for this. Another physical curiosity is the half-integer spin of all fermions and their antiparticles, including the neutron, which has all the properties of a classical angular momentum. But because of its half-integer spin it cannot be explained in a classic way by rotary motion.

Above, both proton and electron were described as the smallest possible and persistent spinning gyroscopes in the quantum world with a four-dimensional diameter $2 \lambda_{w}$, but a different three-dimensional structure that determines the inertial mass. The same must apply to their antiparticles and the neutron and its antiparticle, as inevitably results from the equality of spins in all three fermions. It is known that the fermion spin can assume the sub-values $\hbar / 2$ and $-\hbar / 2$ with respect to any direction and that those spins can take on an odd multiple of $\hbar / 2$, namely $\hbar / 2,3 \hbar / 2,5 \hbar / 2$, etc., where $\hbar=h / 2 \pi$. Evidently the unit $\hbar=$ $h / 2 \pi$ forms the basic rule of the spin to a certain extent. It is known that this angular momentum cannot be traced back to a rotating object, which itself consists of revolving, non-rotating material particles, but must be an intrinsic property of the particles to which we now turn.

With the rotation diameter $2 \lambda_{w}$ of the fermions and $h=\lambda_{w}^{2}$ in the simplest case the four-dimensional angular momentum (spin) takes the value $s=\hbar / 2=h /(2 \times 2 \pi)=\lambda_{w}^{2} /(2 \times 2 \pi)$ at the zero point of the three-dimensional embedding of $\lambda_{w}^{2} /(2 \times 2 \pi)$. The angular momentum in the spatially four-dimensional $\mathrm{R}^{4}$ would therefore be $2 \pi s=\lambda_{w}^{2} / 2=2 \lambda_{w}^{2} / 4$, so that $\lambda_{w}^{2} / 4$ rotates on both sides of the zero point in four-dimensional. However, since according to the above length $\lambda_{w}$ is retained on both sides of the zero point, both surfaces $\lambda_{w}^{2} / 4$ must be triangular surfaces with a common axis $\lambda_{w}=2 \lambda_{x} \equiv 2 \lambda_{1}$. Thus, the four-dimensional angular momentum $2 \pi s$ can only be perceived as point $s=\lambda_{w}^{2} / 4 \pi=\hbar / 2$ in three-dimensional space-time $\mathrm{R}^{3}$.

From the fact that the fermions proton and electron as well as their antibodies are the smallest four-dimensional permanently stable rotational bodies, it follows immediately, that two full rotations of 360 degrees each $\left(2 \times 360^{\circ}\right)$ are required when the rotational axis is rotated to reach the initial value again.

This doubling of the rotation of an axis of rotation in the four-dimensional, which appears strange in three dimensions, is a compelling mathematical consequence. Mathematicians proved, that symmetric rotation in $\mathrm{R}^{4}$ space has two invariant axis-planes which are completely orthogonal, intersect at a point, are left invariant by the rotation and return after rotation by twice the angle around the axis $\left(2 \times 360^{\circ}\right)$. These mathematical-physical facts on the quantum level are therefore also compelling evidence that the three-dimensional space of our world is embedded in a four-dimensional space (see Chapter 5.)

The four-dimensional structure of the fermions also explains the fact that the amount of spin is always the same, while its axis direction can change in a strange way. This alternating spin direction between zero ( 0 ) and one (1) results because, as described above, the four-dimensional surfaces $\lambda_{w}^{2} /(2 \times 2 \pi)$ on both sides of the zero point of the three-dimensional embedding $\lambda_{1} \times \lambda_{2} \times \lambda_{3}$ rotate rigidly in $\mathrm{R}^{4}$, whereas the $\mathrm{R}^{3}$ - embedding with $2 \times \lambda_{1}$ as the axes of rotation in $\mathrm{R}^{3}$ freely movable is. In this way, the axes of rotation can be freely pivoted around the zero point, so that in the simplest cases, $\lambda_{1}$ parallel to $\lambda_{w}$, $\lambda_{1}$ perpendicular to $\lambda_{w}$ and $\lambda_{1}$ inclined by $45^{\circ}$ to $\lambda_{w}$, the spin Figures $1-\cos 0^{\circ}=0,1-\cos 90^{\circ}=1$ and $1-\cos 45^{\circ}=$ $0.29 \ldots$ on the sphere $4 \pi \lambda_{1}^{2}$ (Gaussian sphere). This also shows that the spin behaviors of the fermions and the associated probability calculations in the Hilbert space have a causal physical cause. 


\subsection{Some Physical Applications of Quantum Geometry}

In ref. [15] has been demonstrated, on quantum scale of the space of the three-sphere $S^{3}$ locally each dimension or manifold to be associated with a fundamental length, namely $\lambda_{1}=1 / 2 \times \sqrt{h}$ in $R^{1}$, $\lambda_{2}=\sqrt{2} \times \lambda_{1}$ in $\mathrm{R}^{2}, \quad \lambda_{3}=\sqrt{3} \times \lambda_{1}$ in $\mathrm{R}^{3}$ and $\lambda_{w} \equiv \lambda_{4}=2 \times \lambda_{1}$ in $\mathrm{R}^{4}$ so that must be valid:

$$
2 \lambda_{1}=\sqrt{2} \lambda_{2}=\frac{2 \lambda_{3}}{\sqrt{3}}=\lambda_{w}=\sqrt{h}
$$

These fundamental lengths really seem to constitute the very limit spatially of nature on Euclidian microscopic scale. Also has been proposed the following variations of the latter fundamental lengths to owe the so-called anomalous conductance features in two-dimensional quantum point contact experiments $[17,18]$ :

$$
\begin{gathered}
e^{2} / \lambda_{1}^{2}=4 e^{2} / h=\mathbf{2 . 0 0} \times 2 e^{2} / h \\
e^{2} / \lambda_{1} \lambda_{2}=2 \times 2 e^{2} /(h \sqrt{2})=\mathbf{1 . 4 1} \times 2 e^{2} / h, \\
e^{2} / \lambda_{1} \lambda_{3}=2 \times 2 e^{2} /(h \sqrt{3})=\mathbf{1 . 1 5} \times 2 e^{2} / h, \\
e^{2} / \lambda_{1} \lambda_{4}=2 e^{2} / h=\mathbf{1 . 0 0} \times 2 e^{2} / h, \\
e^{2} / \lambda_{2}^{2}=2 \times 2 e^{2} /(2 h)=\mathbf{1 . 0 0} \times 2 e^{2} / h, \\
e^{2} / \lambda_{2} \lambda_{3}=2 \times 2 e^{2} /(h \sqrt{6})=\mathbf{0 . 8 2} \times 2 e^{2} / h, \\
e^{2} / \lambda_{2} \lambda_{4}=2 e^{2} /(h \sqrt{2})=\mathbf{0 . 7 0} \times 2 e^{2} / h, \\
e^{2} / \lambda_{3}^{2}=2 \times 2 e^{2} /(3 h)=\mathbf{0 . 6 7} \times 2 e^{2} / h, \\
e^{2} / \lambda_{3} \lambda_{4}=2 e^{2} /(h \sqrt{3})=\mathbf{0 . 5 8} \times 2 e^{2} / h, \\
e^{2} / \lambda_{4}^{2}=e^{2} /(h)=\mathbf{0 . 5 0} \times 2 e^{2} / h .
\end{gathered}
$$

Thus, the normal integer conductance plateaus

$$
G=n \frac{e^{2}}{\lambda_{2}^{2}}=n \frac{2 e^{2}}{h}
$$

are integer multiples of the right-hand members of the above listed respective ratios. The "anomalous" conductance plateaus e.g. $G \approx 0.5 \times 2 e^{2} / h, G \approx 0.7 \times 2 e^{2} / h, G \approx 0.85 \times 2 e^{2} / h$ and $G \approx 1.42 \times 2 \mathrm{e}^{2} / h$, respectively, then result from the above variations of the quanta of dimension-dependent lengths in dependence on the respective experimental condition. Clearly all "anomalous" conductance features show up. Even the observed variation of the actual position of the 0.7 feature [19] between 0.6 and 0.8 is neatly explained by the narrow neighborhood of the $\mathbf{0 . 5 8}, \mathbf{0 . 6 7}, 0.7$ and $\mathbf{0 . 8 2}$ structures, respectively, to the former. Furthermore, from this novel microphysical notion results the "additional" factor of two to $e^{2} / h$ quite unconstrained as a consequence of quantum-geometry without any recourse to a special physical model.

In accord with this physical notion the so-called half-plateaus, $G=(n+1 / 2) \times 2 e^{2} / h$ come into existence if $\lambda_{3}$ (instead of $\lambda_{4}$ ) is unity so that $\lambda_{i i i}=1$ and it must be valid $\lambda_{i}=\sqrt{h / 3}$, implying that the above Coulomb formula $e^{2} / \lambda^{2}$ for the first conductance step has to be written in the form

$$
\frac{e^{2}}{\lambda_{i}^{2}}=\frac{3 e^{2}}{h}=1.5 \times \frac{2 e^{2}}{h}
$$

If all further conductance steps switch to the above normal mode, then we receive from the latter Eq- 
uation $(n+1 / 2) \times 2 e^{2} / h$ and, therewith, the conductance half-plateaus.

The quantum-geometrical picture also offers a convincing explanation of the expression for calculating the magnetic flux quantum, i.e. the quantum of magnetic flux passing through a superconductor, given by

$$
\phi_{0}=n \times \frac{\lambda_{2}^{2}}{e}=n \times \frac{h}{2 e}
$$

Furthermore, the puzzling properties of the integer quantum Hall effect (von Klitzing 1980 [20])

$$
R_{H}=\frac{R_{H(n=1)}}{n}=\frac{\lambda_{4}^{2}}{e^{2} \times n}=\frac{h}{e^{2} \times n}
$$

are fully explained as well as the fractional quantum Hall-effect (Strömer, Hill 1984 [21]), where $n=$ $m /(2 m+1)$ and $n=1-m /(2 m+1)=(m+1) /(2 m+1)$ and we, owing to the particular physical situation, obviously observe a fractionalization of $\lambda_{4}^{2}$ such that only the sum of the fractions' reciprocals results again in $\left(\lambda_{4}^{2}\right)^{-1}$, namely:

$$
R_{H(m)}^{-1}+R_{H(m+1)}^{-1}=\frac{e^{2} m}{\lambda_{4}^{2}(2 m+1)}+\frac{e^{2}(m+1)}{\lambda_{4}^{2}(2 m+1)}=\frac{e^{2}}{\lambda_{4}^{2}}=\frac{e^{2}}{h}
$$

I.e. the sum of these fractionally quantized Hall resistances remains constant. In this quantum-geometrical notion the fractional quantum Hall-effect observed in the new material graphen in steps of $n=4(m+1 / 2)$ with the lowest plateau $h /\left(2 e^{2}\right)$ even at room temperature, i.e. $\approx 20^{\circ} \mathrm{C}$ (Novoselov 2004 [22]), is just a special case of the integer quantum Hall effect. It owes its existence to the fact that at the particular experimental conditions the first step of the Hall resistance is not $\lambda_{4}^{2}$ but rather $\lambda_{2}^{2}=1 / 2 h$, where $m=0,1,2, \cdots, m$. Therefore it is clear that the above resistance formula really is valid with $n=4(m+$ $1 / 2)$.

\section{VARIANCE (DIFFERENCE) OF DIMENSION IMPLIES AMBIGUITY OF MAPPINGS IN QUANTUM PHYSICS}

\subsection{Mathematical Evidence}

1) Already in 1878 the mathematician and initiator of set theory, Georg Cantor, showed the mapping to be unique from a line segment into a square, unique from a square into a line segment, i.e. bijective and discrete [23]. With other words: Cantor proved that unique, one-to-one mappings can be constructed between spaces (manifolds) of different dimensions.

2) Whereas his colleague Giuseppe Peano in 1890 especially proved, the mapping firstly to be unique from a line segment into a square, secondly ambiguous from a square into a line segment, i.e. a point in the square generally corresponds to various points in the line segment, and thirdly continuous [24].

3) The final result of this mathematical research has been the widely known theorem of the invariance of dimension, which first has been proven by Luitzen Egbertus Jan Brouwer in 1911 [25]. According to Brouwer's proof can a piece of a k-dimensional manifold bijective and absolute continuously mapped only again into a piece of a $\mathrm{k}$-dimensional manifold, but never into a piece of an m-dimensional one if $m \neq k$. It should be remarked that there have been given many other proofs of the theorem since.

\subsection{Spontaneously Emitted and Propagating Material Particles and Photons Follow Cantor's Mapping Rules, Implying Classical Mechanics to Be Valid for Their Trajectories}

Bohr remarked (see Chapter 4.3.) that the wave behavior in quantum physics only occurs in very special physical situations or constellations (experiments), but that mostly the classical notion of physics is observable. Possibly, this Janus-faced side of quantic nature might be the very reason of Bohr's vague con- 
cept of complementary.

The "normal" undisturbed trajectory of a stable particle or photon, travelling through geometrically three-dimensional space-time, starts with spontaneous emission from four-dimensionality and ends with reception at a location in space-time. I.e. the latter will be recorded by three coordinates of space and one time coordinate. Thus, this topological mapping $V_{4} \in \mathrm{R}^{4} \rightarrow V_{3} \in \mathrm{R}^{3}$ is a point to point mapping but where the way of the mappings carrier, i.e. its trajectory, between the manifolds according to Cantor is discrete: Normally, in nature time and direction in space of the spontaneous emission are non-predictable physically.

Hence, Cantor's mapping law seems to be responsible for physical mappings from $\mathrm{R}^{4}$ into $\mathrm{R}^{3}$ in those cases where the undisturbed trajectories of all particles as carriers of the mappings in space-time, whether with or without rest mass, follow Cantor's rules from their spontaneous emission till reception. Accordingly the propagation of microscopic material particles in space must be thought of as motion of geometrically smallest possible objects with three-dimensional basis in $\mathrm{R}^{3}$ and the proposed four-dimensional height being associated with the former's rest mass. Photons however are emitted and received by four-dimensional atoms at their respective also four-dimensional Bohr radii (see Chapter 4.3.) and also moving through three-dimensional space in accord with Cantor's mapping rules. This topological-physical notion of particle and photonic trajectories as bijective but discrete mappings $V_{4} \in \mathrm{R}^{4} \rightarrow V_{3} \in \mathrm{R}^{3}$ and, therewith, as classical (including special relativity) inertial translational motion and photonic propagation, respectively, implies its causality. Hence, this kind of mapping is by no way wavelike, neither in the form of Schrödinger's wave-packet nor of the de Broglie wave for single particles or of photons according to Einstein. In so far current paradigms that the propagating particle, whether light or material, is either a pure wave-packet (e.g. Schrödinger) or some crossing of wave and particle (e.g. the early de Broglie) are likewise not true physical notions. This also implies that the entanglement of quantum systems in the case of mappings according to Cantor does not exist (nor to Peano; see below). The latter non-existence of entanglement also must be valid in the case of the widely discussed correlation of physical properties of two or more particles (photons) separated by any given distance. In all those cases also a Peano ambiguity cannot occur (see below). Therefore, if a physical property $a_{1}$ of a quantum system $S_{1}$ is measured (recorded in $\mathrm{R}^{3}$ ), then the value $\mathrm{a}_{2}$ of the correlated physical property of the separated system $\mathrm{S}_{2}$ automatically follows. I.e. from the non-existence of entanglement in the sense of current theory automatically follows non-existence of instantaneous non-local collapse of the putative wave-function. Einstein's reproved spooky superluminal action at a distance is not a realistic physical notion in the case of physical Cantor mapping but neither in Peano mappings, as will be shown in the following chapters. Also will be demonstrated, the very recently published "disproof of theories based on classical trajectories" not to discriminate the preceding, i.e. the physical existence of classical trajectories of material particles and photons in the case of Cantor mappings.

\subsection{Peano's Ambiguity of Mappings between Manifolds with Difference of Dimension Equal to One Explains the Wavelike Behavior of Material Particles and Photons}

Richard Feynman: "It will take just this one experiment, which has been designed to contain all of the mystery of quantum mechanics, to put you up against the paradoxes and mysteries and peculiarities of nature one hundred per cent. Any other situation in quantum mechanics, it turns out, can always be explained by saying, You remember the case of the experiment with the two holes? It's the same thing."

Note, that in all quantum-mechanical and optical experiments, where the wavelike distribution of particles and photons becomes manifest, neither the physical built-up of the respective particle(s) is measured nor its above discussed undisturbed trajectory in the course of Cantor mapping, but rather e.g. position (coordinates), momentum, time or spin, i.e. exclusively physical properties, recorded after the Cantor trajectory's interruption in nature or by experiment. This will say, that the wave pattern only occurs if the classical trajectory's Cantor mapping is interrupted through e.g. scattering, the passage of a measuring device, a crystalline structure or the edge of some material, or, if an electron orbiting about the atomic nucleus is considered and such those physical phenomena are brought into contact with the latter's 
four-dimensional topology. Of course, these are only paraphrases of the most popular physical phenomena in the quantum domain, namely e.g. scattering, diffraction of particles and photons by a double slit, of photons and particles by a crystal and of particles and photons passing the edge of some material about in a distance analogous to the width of the slits in the double-slit experiments and the generation of the atomic spectra.

We maintain that in all those cases, and others more (e.g. Chapter 4.4.), we directly observe the four-dimensional structure of material particles and, in association with this, Peano's ambiguity of topological mappings through physical carriers (material particles and photons) from $\mathrm{R}^{4}$ into the spatially three-dimensional space-time $\mathrm{R}^{3}$. From the preceding and the history of quantum physics it is obvious, that it will suffice to show, the de Broglie wave as well as Heisenberg's uncertainty principle directly to follow from the latter suppositions. This means, that especially the de Broglie wave directly expresses the magnitude of ambiguity of a mapping from $\mathrm{R}^{4}$ into $\mathrm{R}^{3}$ in dependence on the momentum of a single particle as carrier of the mapping.

Thus, in association with the preceding discussion referring to the spatially four-dimensional built-up of fermionic matter as well as the of the Universe, Peano's proof seems to be of special physical interest although with a scale difference of

$$
\frac{P}{2 \lambda_{w}} \equiv \frac{P}{2 \sqrt{h}}=c_{A}^{4}(1-\sin A) \cos ^{5} A=4.88 \times 10^{40}
$$

We consider a mapping from spatial dimension $k(=4)$ into dimension $k-1(=3)$, abstract from dimensions $k-2$ and, then, in any case Peano's finding can directly be applied such that, according to the preceding, continuity of the mapping is postulated with the consequence, that a point in volume $\mathrm{V}^{k}\left(\mathrm{~V}^{4}\right)$ corresponds to various points in $\mathrm{V}^{k-1}\left(\mathrm{~V}^{3}\right)$ and the bijection of the mapping, i.e. one-to-one correspondence, gets lost, implying its ambiguity. In the case of material particles we proceed for reasons of simplicity from a single proton as the carrier of the topological mapping. By rewriting the de Broglie formula $\lambda_{B}=$ $h \cdot p^{-1}$ of the moving proton, with rest mass $m_{0}$, we receive in the relativistic borderline case, where the Lorentz factor $\gamma \approx 1$, in association with Equation (2):

$$
\lambda_{B} \times m_{0} v=h \equiv \lambda_{w}^{2}
$$

Factor $2(1-\sin A)$ in Equation (2) is neglected. We rewrite this expression into

$$
\lambda_{B} \times \frac{\lambda_{w} v}{c}=\lambda_{w}^{2}
$$

Obviously is the latter formula the physical transformation of Peano's above introduced topological mapping from (four-dimensional) plane $\lambda_{w}^{2}$ as the product of the (four-dimensional) line segment $\lambda_{w} v / c=\tau_{w}=m_{0}$ and the (three-dimensional) ambiguous line segment $\lambda_{B}=\lambda_{w} \mathcal{C} / v$, such that really applies:

$$
\lambda_{B}=\frac{\lambda_{w}^{2} c}{\lambda_{w} v}=\frac{\lambda_{w}^{2}}{\tau_{w} v}=\frac{h}{p}
$$

If any particle with mass $m<m_{0}$ is considered, then $\lambda_{w}$ in the left-hand side of Equation (26) and in the denominator in the middle of Equation (27) becomes $n \times \lambda_{w} v / c$, where $n=m / m_{0}$, because only for the proton $\lambda_{1} \lambda_{2} \lambda_{3}=1$ is valid. From this is obvious that not the mass of the mapping's carrier be the decisive factor but the momentum $p=\lambda_{w} n v / c=m v / c$. Now, if a proton with dimensionless momentum $p=\lambda_{w} v / c$ according to Equation (26) approaches another material object with four-dimensional height $\lambda_{w}$ then, at three-dimensional distance $\lambda_{w} \mathcal{C} v$, one obtains

$$
\frac{\lambda_{w} C}{v} \times \frac{\lambda_{w} v}{C}=\lambda_{B} \times \frac{\lambda_{w} v}{C}=\lambda_{w}^{2}=h
$$

Thus, from this (four-) two-dimensional plane $\lambda_{w} v / c \times \lambda_{w} c / v=\lambda_{w}^{2}$ a Peano mapping into (three) one-dimensional line segment $\lambda_{w} c l v$ with ambiguity $\lambda_{B}$ in accord with Equations (26) and (27) is received. 
Note that Equation (28) can also be rewritten as:

$$
T_{w} \times T_{w} c^{2}=\lambda_{w}^{2}, \lambda_{B(T)}=\frac{\lambda_{w}^{2}}{\tau_{w} c^{2}}=\frac{h}{E_{p}}
$$

This means, that the already experimentally known quantum geometric effect, dubbed tunneling effect, follows also directly as ambiguity effect in accordance with the above.

It is proposed, this topological effect to be the very cause of all known quantum-physical phenomena, especially of the above mentioned interference of material particles (and photons; see below) by a double slit and diffraction by traversing a crystal or passing the edge of some material about in a distance analogous to the width of the slits in the double-slit experiments, furthermore, of the generation of the atomic spectra. In the following especially the physical phenomena referring to the double-slit experiments and the origin of the Bohr-radius' wave-structure shortly shall be discussed whereby, as widely known, in the former case three physical situations are to distinguish: firstly the double-slits shall be open simultaneously, secondly the slits shall be open successively and third the double-slits shall be open simultaneously with detectors behind the slits.

It is obvious that according to Peano's proof every quantum-mechanical event in $\mathrm{R}^{4}$ occurs at a unique four-dimensional point (coordinates), which in $\mathrm{R}^{3}$ only ambiguously can be recorded with likewise ambiguous coordinates if the unique Cantor trajectory is interrupted through the above discussed physical situations where the four-dimensional structure of matter plays the decisive role. Thus, topologically two open slits for approaching and the slits passing particles according to Equation (28) in association with Equation (27) on passing will deliver two parallel ambiguous mappings $\lambda_{B}$ implying the generation of an interference pattern of two ambiguity amplitudes according to wave physics. One should notice that, whilst approaching one slit according to Equation (28), the particle's ambiguity with respect to the other slit must be

$$
\lambda_{B}^{\prime}=\sqrt{\frac{\lambda_{w}^{2} c^{2}}{v^{2}}+d^{2}}=\frac{\lambda_{w} c}{v} \sqrt{1+\frac{d^{2} v^{2}}{\lambda_{w}^{2} c^{2}}}
$$

where $d$ denotes distance between the two open slits, such that for the ambiguity ratio of the open slits referring to one single incident particle applies

$$
\frac{\lambda_{B}^{\prime}}{\lambda_{B}}=\sqrt{1+\frac{d^{2} v^{2}}{\lambda_{w}^{2} c^{2}}}
$$

This implies that for all particles, directly approaching one slit, according to Equation (28) the probability exists of value

$$
P_{B}^{\prime}=\left(1+\frac{d^{2} v^{2}}{\lambda_{w}^{2} c^{2}}\right)^{-1 / 2}
$$

to eventually pass the other slit such that, referenced to one slit, the total probability is the sum

$$
P_{B}=\left(1-P_{B}^{\prime}\right)+P_{B}^{\prime}=\left[1-\left(1+\frac{d^{2} v^{2}}{\lambda_{w}^{2} c^{2}}\right)^{-1 / 2}\right]+\left(1+\frac{d^{2} v^{2}}{\lambda_{w}^{2} c^{2}}\right)^{-1 / 2}=1
$$

This implies occurrence of the well-known two-slit interference pattern of the two ambiguity waves at the receiver behind the slits even if only one particle (photon) is involved (see below). Whereas according to Equations (28) and (27) with only one slit open, only one ambiguous mapping $\lambda_{B}$ and, therewith, the normal single-slit diffraction pattern necessarily must occur. Obviously ratios (31) and (32) can be used to test the proposed theory by varying distance $d$.

Note, that a recent double-slit-diffraction experiment, which recorded single electron detection events 
diffracting through a double-slit, with the result that a diffraction pattern was built up from individual events, exactly complies with the preceding theoretical expectations [26] (see below).

In the third case, with detection of the incident particle, in what form ever, after passing the slits, are the three-dimensional coordinates of both particles uniquely recorded in $\mathrm{R}^{3}$ (space-time) and, therewith, ambiguity $\lambda_{B}$ in all cases terminated such that we now have bijective mapping $V_{3} \in \mathrm{R}^{3} \rightarrow V_{3}^{\prime} \in \mathrm{R}^{3}$, implying classicality, such, that diffraction and, therewith, an interference pattern cannot occur at reception. Note the similarity of the latter topological termination of the ambiguity wave $\lambda_{B}$ with Bohr's otherwise physically unfounded assumption, that observation (measuring) causes the collapse of the wave. I.e., according to Kopenhagen follows from the intended action by itself (per se) non-observation of the wave, with the conjectured spooky (Einstein) consequences (see Chapter 4.4.). It is clear, that in the latter case all forms of the above discussed interference pattern will revive, if after recording the three-dimensional coordinates both particles again would undergo a physical situation as described in Equation (28) and the following.

Besides, if we require Heisenberg's uncertainty relation for an approaching proton,

$$
\Delta x \times \Delta p \geq h
$$

to take the form

$$
\Delta x \times \Delta p=h
$$

then, according to the above, this can be rewritten into

$$
\Delta x \times \Delta p=\lambda_{B} \times m_{0} v=\frac{\lambda_{w} c}{v} \times \frac{\lambda_{w} v}{c}=\lambda_{w}^{2}=h
$$

I.e. $\Delta x=\lambda_{B}$ and $\Delta p=m_{0} v$, because on this elementary level protonic mass must be $m_{0}=\lambda_{w} / c$. Clearly relation (35) in association with (33) contradicts Equations (25) to (27) if $p=m_{0} v$ increases and $\lambda_{B}$ equivalently decreases, with $h=\lambda_{w}^{2}$ remaining constant. This also is true if we switch to the relativistic version, with non-negligible Lorentz factor $\gamma>1$ :

$$
\Delta x \times \Delta p=\lambda_{B} \times m_{0} v=h \gamma^{-1}
$$

Thus, it follows the uncertainty relation (23) to be the relativistic version of the de Broglie Equation (15) such that applies

$$
\Delta x \times \Delta p=\lambda_{B} \times m_{0} v \times \gamma \geq h
$$

Heisenberg was of the opinion that it is not possible to measure energy and time as precisely as desired and therefore "physics should only formally describe the context of the perceptions", furthermore, "because all experiments are based on the laws of quantum mechanics and thus the Equation (33) are subject, then quantum mechanics definitively establishes the invalidity of the causal law."

According to the above, Heisenberg's "measurements" are of no importance at all. They can only ever be accurate to a certain extent. Whereas the topological effects described above are conditioned by natural laws and therefore completely independent of any measurements. Therefore, in any case the physical terms "time" and "impulse" are not arbitrarily precise in nature, but always "sharp". In particular, this applies to the time quantum $\tau_{w}$ and thus for any period of time anyway, but the impulse and speed are also always "sharp" in terms of direction and speed. In all cases, wave phenomena are only triggered, when quantum objects come so close that their four-dimensionality triggers the effects described above. These are completely causal, but not directly traceable due to the Peano's mapping ambiguity between $\mathrm{R}^{3}$ and $\mathrm{R}^{4}$.

In the case of a single photon we proceed from Einstein's equation of the photon energy

$$
E_{\text {phot }}=m_{\text {phot }} c^{2}=h v=h c / \lambda_{\text {phot }}=\lambda_{w}^{2} c / \lambda_{\text {phot }} \text { by rewriting the latter formula to }
$$

$$
\lambda_{\text {phot }} \times m_{\text {phot }} c=\lambda_{\text {phot }} \times \Delta \tau_{w} c=\lambda_{w}^{2}
$$

since according to Equation (2), equivalently must be valid $m_{p h o t}=\Delta \lambda_{w} / c=\Delta \tau_{w}$. Hence, in the case of the 
photon as physical carrier of Peano's topological mapping, we obviously have the ambiguous mapping from two-dimensional plane $\lambda_{w}^{2}$ into one-dimensional line segment $\Delta \lambda_{w}=\lambda_{w} \tau_{w} / \Delta \tau_{w}$ with Peano ambiguity $\lambda_{\text {phot }}$.

If analogously to the foregoing a photon with momentum $\Delta \tau_{w} c$ according to Equation (38) approaches a material object on quantum scale, then at distance $\lambda_{w} \tau_{w} / \Delta \tau_{w}$ we have $\Delta \tau_{w} c \times \lambda_{w} \tau_{w} / \Delta \tau_{w}=\lambda_{w}^{2}=h$ and, thus, from this two-dimensional plane, also a Peano mapping into ambiguous one-dimensional $\lambda_{w} \tau_{w} / \Delta \tau_{w}=$ $\lambda_{p h o t}$ in accord with Equation (38), being the basic topological cause of all known wavelike phenomena of light. From the preceding one derives the propagating photon's "frequency" and energy to be of value:

$$
v=\frac{\Delta \tau_{w}}{\tau_{w}^{2}}=\frac{\Delta \tau_{w} c^{2}}{h}, E_{p h o t}=\Delta \tau_{w} c^{2}
$$

Notice the analogous physical built-up of the proton's rest energy $E=\lambda_{w} \mathcal{C}=\tau_{w} \mathcal{C}^{2}$ and of the photon's energy $E_{\text {phot }}=\Delta \lambda_{w} \mathcal{C}=\Delta \tau_{w} \mathcal{C}^{2}$, respectively.

Of course, the latter reasoning could have been abridged through referring to de Broglie's above mentioned introduction of the wave-concept for material particles as analogue to the wave behavior of single photons.

Finally it will be shown that the increase of "waves" in steps of $n=1,2, \cdots, n$ of the by Bohr assumed electron's circular orbit in the case of the hydronic atom, such that $\lambda_{B}=2 \pi r_{n} / n$, solely is due to the previously discussed quantization in steps of elementary length $\lambda_{w}$. We rewrite Equation (25) in accord with Equation (23) to

$$
\lambda_{B}=\frac{h}{m_{e} v_{e}}=\frac{\lambda_{w}^{2} k}{2 \tau_{w}(1-\sin A) v_{e}}=\lambda_{w} \frac{k c}{2(1-\sin A) v_{e}}
$$

where $2 \tau_{w}(1-\sin A) / k=m_{e}$. Through insertion of $v_{e}=h /\left(2 \pi m_{e} r_{1}\right)$ into the second left-hand member of the latter eq. we receive $\lambda_{B}=2 \pi r_{1}$ and hence

$$
\lambda_{B}=\lambda_{w} \frac{k c}{2(1-\sin A) v_{e}}=2 \pi r_{1}
$$

where $r_{1}$ denotes first Bohr-radius. The latter mid-ratio can be extended in steps of $n / V_{e}$ only, so that speed $V_{e}$ decreases accordingly, since $\lambda_{w}$ must be conserved, such that for any radius applies:

$$
\lambda_{B(n)}=n \lambda_{w} \frac{k c}{2(1-\sin A) v_{e}}=2 \pi r_{n}
$$

I.e. the circumference of the Peano-ambiguities of the four-dimensional Bohr-radii is an integer multiple of the de Broglie wave due to quantization in steps of value $\lambda_{w}$. As before, it is obvious that not a real wave is associated with the orbiting electron, nor is the Peano-de Broglie ambiguity rotating about the nucleus. Rather, the electron's whole four-dimensional orbit ambiguously is hidden for the $\mathrm{R}^{3}$-observer.

Eventually it should be emphasized that by inversion of the preceding topological argumentation, from the quantum-mechanical experimental findings conclusively follows the four-dimensionality of the micro cosmos to be the simplest and most plausible interpretation.

According to the previous it is also obvious that due to the fact that $\lambda_{w}$ intersects local three-dimensional XYZ-plane $\left(\lambda_{1} \lambda_{2} \lambda_{3}\right.$ on elementary scale) of the three-sphere at zero-dimensional point (singularity), rest time and rest mass (rest energy) must be scalars which always take well determined values in the rest frame, implying that they cannot be subject of quantum mechanical rules, as already remarked by Dirac and Feynman as "sharpness".

Thus, the conclusion is unavoidable: The ambiguity of physical-topological Peano-mappings $V_{4} \in \mathrm{R}^{4} \rightarrow V_{3} \in \mathrm{R}^{3}$ is not associated with a real wave or wave-packet propagating in spacetime, with real group velocity, phase speed, momentum and energy of the wave. Rather it is a "stationary" topological phenomenon, bound solely to the previously discussed and many more natural or experimental physical 
situations, where a mapping through a material or photonic carrier from the four-dimensional structure of matter into spatially three-dimensional space-time becomes manifest. This implies that the Schrödinger equation and related wave-functions are specialized mathematical models of the Peano ambiguity of accumulations of material and photonic carriers or of physical properties of the latter in four-dimensionality, as e.g. the spin. I.e. wave-functions are mathematical devices or computational tools with no real physical significance. This obviously implies non-existence of entanglement in the sense of current theory and of the associated instantaneous non-local collapse of the respective wave-function (Einstein's "spooky action at a distance"), even in cases where this apparently seems to be proven. Rather, it can be assumed that two entangled but known quantum states are more or less "frozen" and that they keep this state when separated, so that even after a considerable distance, when one state is measured, that of the other immediately follows. In other words, each of these two quantum states, which are now separated, is not uncertain, but rather certain, though unknown, so that when one of the two states is disclosed (observation, measurement) that of the other results immediately. This applies to any distance, no matter how long. A "spooky action" (Einstein) with a high speed incredibly faster than light is therefore not required.

Finally is to stress: The fact that already a single quantum carrier linearly triggers topological ambiguity in the form of the de Broglie-wave for material particles and Einstein's equation for photons, respectively, is obviously the very cause of the algebraic linearity of the wave-functions as summations of many single linear ambiguity events.

\subsection{Apparent Experimental Proof and Disproof of Nonlocal Wave-Function Collapse of a Single Particle and of the Classicality of Trajectories, Respectively}

E.g., consider the above mentioned "Experimental proof of nonlocal wave-function collapse for a single particle using homodyne measurements" [27].

The authors claim for the first time to have demonstrated the instantaneous non-local collapse of the wave-function of a single photon by splitting the latter photon by means of a beam splitter of adjustable reflectivity $\mathrm{R}$ (reflection and deflection) between two laboratories and experimentally testing if the choice of measurement in one lab really causes a change in the local quantum state in the other lab. This for they have been using homodyne measurements with some different measurement settings. Notice that the authors maintain to have been splitting a single photon (into two portions of the single photon), the entanglement of the photon's splits and the non-local collapse of the wave-function.

According to the preceding not a photon has been split but Peano's ambiguity wave, according to Equations (15) to (17), when the incident photon approached the beam splitter according to Equation (28). Hence, it must be valid

$$
\lambda_{\text {phot }}=\frac{\lambda_{w} \tau_{w}}{\Delta \tau_{w}}=\lambda_{\text {phot }}^{\prime}+\lambda_{\text {phot }}^{\prime \prime}=\frac{\lambda_{w} \tau_{w}}{\Delta \tau_{w}} R+\frac{\lambda_{w} \tau_{w}}{\Delta \tau_{w}}(1-R)
$$

where $R$ denotes reflectivity as rational number $\leq 1$. According to the preceding, must the probabilities to observe the photon in one or the other lab ambiguously add up as to

$$
P=P^{\prime}+P^{\prime \prime}=R+(1-R)=1
$$

Now in the case of homodyne detection or non-detection of the incident photon in one of the both labs i.e. unique record of its presence or non-presence implying immediate switch to bijective mapping $V_{3} \in \mathrm{R}^{3} \rightarrow V_{3}^{\prime} \in \mathrm{R}^{3}$, associated with termination of ambiguity $\lambda_{\text {phot }}=\lambda_{\text {phot }}^{\prime}+\lambda_{\text {phot }}^{\prime \prime}$, the former ambiguous probability to observe the single photon promptly takes the unique form

$$
P=P^{\prime}+P^{\prime \prime}=1(0)+0(1)=1
$$

It is clear that the photon (particle) analogously to the double slit experiment always is traveling only one definite way, but which, owing to Peano mapping $V_{4} \in \mathrm{R}^{4} \rightarrow V_{3} \in \mathrm{R}^{3}$, ambiguously is hidden for the observer in $\mathrm{R}^{3}$. 
As already mentioned, has recently apparently been proven non-classicality of a cesium atom's motion on a discrete lattice, i.e. a so-called quantum walk [28]. The latter atom moves in one of the two standing optical waves of laser light with $\lambda_{\text {phot }}=866 \mathrm{~nm}$ that have opposite electric-field polarizations. In this connection should be noticed that the photons of laser light, due to the enforced stimulated emission and, hence, non-random (non-discrete) parallel propagation of all photons into one definite direction are no more carriers of discrete and one-to-one Cantor mapping as introduced above, but of ambiguous Peano mapping instead. The experiment consists of measuring correlation between the atom's positions at different times in a complicated manner. The further details are not of interest for our purpose to show that the experiment does not disprove classicality of trajectories, i.e., unique and bijective Cantor mapping, since it really is a further subset of the previously discussed ambiguous Peano mapping. Owing to the atom's enclosure in an ambiguous photonic lattice relative to a resting photonic lattice, the movement of the atom from its rest position in either direction in jumps of one or more wavelengths to another lattice will in the simplest case be ambiguous

$$
\lambda_{B}=\frac{\lambda_{w}^{2}}{m_{C s} v}=\lambda_{B}^{\prime}+\lambda_{B}^{\prime \prime}=\frac{\lambda_{w}^{2}}{2 m_{C s} v}+\frac{\lambda_{w}^{2}}{2 m_{C s} v}
$$

whereby $m_{C s}$ denotes mass of Cesium atom. Thus, e.g., for one jump we have Peano ambiguity of the probability in the order of

$$
P=P^{\prime}+P^{\prime \prime}=\frac{1}{2}+\frac{1}{2}=1
$$

After measuring, i.e. detection or non-detection and associated prompt switch to bijective mapping $V_{3} \in \mathrm{R}^{3} \rightarrow V_{3}^{\prime} \in \mathrm{R}^{3}$, this takes the unique form

$$
P=P^{\prime}+P^{\prime \prime}=1(0)+0(1)=1
$$

Therefore, the experiment's set-up is unsuitable to prove or disprove classicality of trajectories but rather shows that the Peano ambiguity is valid in this very special physical situation too.

The above reasoning, that the photonic enclosure of the cesium atom enforces Peano mapping of its propagation in units of the laser's wavelength, also is directly verifiable. Consider that according to the preceding Equation (28) and (38) it must be valid $\lambda_{B} \times \frac{\lambda_{w} v}{c}=\lambda_{w}^{2}$ and $\lambda_{\text {phot }} \times \Delta \tau_{w} c=\lambda_{w}^{2}$. If one demands $\lambda_{B}=\lambda_{\text {phot }}$ then this implies $\frac{\lambda_{w} v}{c}=\Delta \tau_{w} c=\tau_{w} v$. Therefore, if instead of $\frac{\lambda_{w} v}{c}$ the real cesium atom's dimensionless momentum $p_{C s}=n 2 \lambda_{w}(1-\sin A) v / c$ is introduced, where $n=m_{C S} / m_{0}=131.9454$, then automatically follows $2 \lambda_{B}=n \lambda_{w}(1-\sin A) v / c=\lambda_{\text {phot }}=\Delta \tau_{w} c$, or, $\lambda_{B}=1 / 2 \lambda_{\text {phot }}$, so that can be written:

$$
\lambda_{B}=\frac{\lambda_{\text {phot }}}{2}=\frac{\lambda_{w}^{2}}{m_{C S} v}=\frac{\lambda_{w} c}{2 n(1-\sin A) v}
$$

It is clear that owing to the ambiguity of the atom's location within the ambiguous photonic lattice, the velocity of the "jumps" in steps $\lambda_{B}=1 / 2 \lambda_{\text {phot }}$ in the denominator of the most right-hand side of Equation (49) can be anything between $-1 / 2 \leq v \leq 1$ of the wavelengths in $\mathrm{cm} \cdot \mathrm{s}^{-1}$. For this exists the evolution $\ln (1+v)=v-v^{2} / 2+v^{2} / 3-\cdots$ such that especially is valid $\ln 2=1-1 / 2+1 / 3-\cdots$ and $\mathrm{e}^{v}=\mathrm{e}^{\ln 2}=2$. Thus, the atom's ambiguous velocity (in the standing wave: quantum-walk) must be $V=\ln 2 \mathrm{~cm} \cdot \mathrm{s}^{-1}=0.69315$ $\mathrm{cm} \cdot \mathrm{s}^{-1}$. With the proper values of $v, c, \lambda_{W}$ in $\mathrm{cm}, n$ and angle $A$ inserted into the latter formula we receive

$$
\lambda_{B}=\frac{\lambda_{\text {phot }}}{2}=\frac{0.814013 \times 10^{-13} \times 2.99792 \times 10^{10}}{2 \times 131.9454 \times\left(1-\sin 43.788^{\circ}\right) \times 0.69315}=433.15 \mathrm{~nm}
$$

I.e. nearly exactly the reported $1 / 2 \lambda_{\text {phot }}=433 \mathrm{~nm}$ of the laser light. 


\section{PROPOSED FOUR-DIMENSIONAL TOPOGRAPHY OF THE UNIVERSE AND THE ASSOCIATED AMBIGUITY OF THE COSMIC REDSHIFT}

\subsection{Brief Summary of the Physics within a Four-Dimensional Curved Spinning Universe Also as the Cause of the Redshift of the Light from Far Emitters}

Above has been introduced the proposal the Universe to be an Einsteinian near spherical static rotation hyper ellipsoid spinning in a geometrically four-dimensional space with Euclidian $\mathrm{E}^{4}$ metric [15]. Furthermore, that every observer at any point within the three-sphere all ingoing light from any distance will perceive as tangential Minkowskian pseudo Euclidian projection such that the four-dimensional Euclidian distance spatially is transformed into time associated with apparent recession velocity, apparent time dilation and associated redshift (the latter explains the deviations of distance calculations on the grounds of supernova data from the standard expansion model - in the latter explained through "dark energy" as apparent ones). This implies that any receiver at any point of the three-sphere will perceive the emitter's time to be apparently dilated and, thus, the ingoing light redshifted. The CMB has been explained as Planckian radiation resulting from cyclic red-and blue-shift of all light circling the Universe, and from the former's energy density its correct temperature $2.72 \mathrm{~K}$ has been calculated, where $\mathrm{K}$ denotes Kelvin.

Now, how does red-shift of light in curved space, other than in expanding plane Minkowskian space through the optical Doppler-effect, happen physically? This for, consider a geodesic ingoing at a resting receiver from a far-away emitter in distance $\Delta P \pi / 2$ and, therewith, the ingoing light considerably red-shifted, as we know since the pioneer work of Georges Lemaitre (1927) and Edwin Hubble (1929). According to the law of optical deflection must the light ingoing from curved space for any resting observer be reflected or mapped into a plane pseudo-Euclidian Minkowski space. Since self-motions in the great distances within the hypersphere can be neglected, and the transmitter and receiver can therefore be viewed as fixed relative to one another, it is clear that the measured redshifts cannot be explained by the optical Doppler effect of the prevailing theory (expansion of flat space). Rather it must be due to another hitherto unknown physical effect, to which we now will turn. With Equation (5) the quantum-geometrical origin of time has been derived as the mapping of the four-dimensional radius $\lambda_{w}$ of the proton (electron) in the form of the dimensionless tie-point $-\lambda_{x}^{2}+\lambda_{y}^{2}+\lambda_{z}^{2}-\lambda_{w}^{2}=0$ in $\mathrm{R}^{3}$ as the quantic continuum $\tau_{w}$ or sequence of points $\mathrm{d} \tau_{w}$, with highest possible velocity $\omega_{P} \equiv C=$ const., such that is valid $\mathrm{d} \lambda_{w}=\omega_{P} \mathrm{~d} \tau_{w}$ and so the improper integral Equation (5). The fact that $\lambda_{w}$, and therefore $\tau_{w}$ is always perpendicular to its embedding in $\mathrm{R}^{3}$ means that in the curved space of the Einsteinian hypersphere the time quanta $\tau_{w}$ of the receiver's rest system and the respective time quanta $\tau_{w}^{\prime}$ of a very distant emitter must be inclined one to another in direct relation to the curvature by angle A of the geodesic ingoing at the receiver. However, because according to the above the respective geodesic ingoing at the receiver is mapped as a pseudo-Euclidean Minkowski space, will the zero points of the $\mathrm{R}^{3}$-embedding of $\tau_{w}^{\prime}$ and $\tau_{w}$ come to lie on a Euclidean line. The time quantum $\tau_{w}^{\prime}$ of the distant emitter is thus rotated by the angle $\mathrm{A}$ in relation to the time quantum $\tau_{w}$ of the receiver in the $3 \mathrm{D}$-sphere so that the time quantum $\tau_{w}^{\prime}$ appears stretched analogously to the above special-relativistic Equation (4) and it follows $c / v^{\prime}=c \tau_{w}^{\prime}=\lambda_{o b s}$. Therefore, the geodetic curvature around the angle A must become visible in the receiver's pseudo-Euclidean Minkowski space through extension of the received light's wavelength by the factor:

$$
\lambda_{\text {obs }}=\lambda_{0}\left(1+\frac{\sin A}{\cos A}\right)=\lambda_{0}(1+\tan A)=\lambda_{0}(1+z), \quad z=\tan A
$$

Therefore follows directly:

$$
\frac{\lambda_{o b s}}{\lambda_{0}}=1+z=\frac{1+\frac{v_{a p p}}{c}}{\sqrt{1-\frac{v_{a p p}^{2}}{c^{2}}}}, \quad z=\tan A=\frac{1+\frac{v_{a p p}}{c}}{\sqrt{1-\frac{v_{a p p}^{2}}{c^{2}}}}-1
$$


This implies, $\frac{v_{a p p}}{c}$, the dimensionless velocity derived from the latter redshift, be only an apparent one:

$$
\frac{v_{a p p}}{c}=\frac{(1+z)^{2}-1}{(1+z)^{2}+1}=\frac{(1+\tan A)^{2}-1}{(1+\tan A)^{2}+1}
$$

In this way the fixed four-dimensional distance of the emitter's position within the three-sphere projected into the pseudo-Euclidian Minkowski projection by the ingoing light, at the receiver is transformed into the simulacrum of the emitter's apparent dimensionless recession velocity.

Consequently every receiver within the sphere can the spatial four-dimensional coordinate of the emitter also perceive as pseudo-Minkowskian in the form of dilation of the emitter's time by the Lorentz-factor:

$$
\left(1-\frac{v_{a p p}^{2}}{c^{2}}\right)^{-\frac{1}{2}}=\left\{1-\left[\frac{(1+\tan A)^{2}-1}{(1+\tan A)^{2}+1}\right]^{2}\right\}^{-\frac{1}{2}}
$$

Since in the hypersphere all geodesics can be considered to have the same curvature and all $\lambda_{w}$, and thus $\tau_{w}$, are perpendicular to the zero point of their respective three-dimensional embedding, any geodesic of any length can also be expressed with the same angular dimension as that used in the above equations, namely by the Angle " $A$ ".

\subsection{Geometrically Four-Dimensional Universe Enforces Peano Ambiguity of the Cosmic Redshift}

The universe is assumed here as a rotating Einsteinian hypersphere, but it is clear that the distribution of centrifugal forces within the sphere rather suggests an elliptical shape. Recent observations of different redshifts at roughly the same cosmic distances e.g. would do this for redshifts in a hyper-ellipsoid. From Equation (53) follows

$$
\frac{(1+z)^{2}-1}{(1+z)^{2}+1}=\frac{v_{a p p}}{c}=\frac{2 r_{A}}{P \pi}
$$

where $r_{A}$ denotes apparent distance in tangential Minkowski projection on the grounds of the dimensionless apparent recession velocity $V_{a p p} / c$. This delivers the constant value $v_{a p p} / r_{A}=2 c /(\pi P)=$ const. The latter relation enables one to calculate radius $P$ and, therewith, the circumference of the three-sphere $S^{3}$ by considering that, to mention it again, $V_{a p p}$ denotes apparent recession velocity per distance $r$ of $1 / 4 \sigma=1 / 2 \pi P$, which is identified as the Hubble constant $H_{0} \equiv v_{a p p} / r_{A}=2 c /(\pi P)=$ constant, wherefrom applies

$$
P=\frac{2 c}{\pi H_{0}}
$$

Independent measurements of the cosmic parameter $H_{0}$ have all led to different values, the highest value being $H_{0} \approx \geq 74 \mathrm{~km} \cdot \mathrm{s}^{-1} \cdot \mathrm{Mpc}^{-1}$ and the lowest $\approx 67 \mathrm{~km} \cdot \mathrm{s}^{-1} \cdot \mathrm{Mpc}^{-1}$, where $\mathrm{km}$ denotes kilometer, $\mathrm{s}$ second and Mpc Mega parsec. The values $\approx \geq 74 \mathrm{~km} \cdot \mathrm{s}^{-1} \cdot \mathrm{Mpc}^{-1}$ were all obtained with space telescopes, whereas $H_{0} \approx 67 \mathrm{~km} \cdot \mathrm{s}^{-1} \cdot \mathrm{Mpc}^{-1}$ from the Planck satellite on the basis of repeated measurements of the "local" CMB radiation and by earth-bound telescopes was determined. Experts consider $H_{0} \approx 67 \mathrm{~km} \cdot \mathrm{s}^{-1} \cdot \mathrm{Mpc}^{-1}$ as the correct value, while highest $H_{0} \approx \geq 74 \mathrm{~km} \cdot \mathrm{s}^{-1} \cdot \mathrm{Mpc}^{-1}$ is controversial. Based on the above Equation (15) a relativistic solution of the discrepancy has been proposed, with the result that $H_{0} \approx 67 \mathrm{~km} \cdot \mathrm{s}^{-1} \cdot \mathrm{Mpc}^{-1}$ from the Planck satellite is correct, whereas the measurements of the space telescopes are relativistically distorted [29]. But because the most observations and therefore also most numbers in the literature stem 
from space telescopes, the value $H_{0}=75 \mathrm{~km} \cdot \mathrm{s}^{-1} \cdot \mathrm{Mpc}^{-1}$ is chosen as the basis for the following calculations. With $H_{0}=V_{a p p} / r_{A}=75 \mathrm{~km} \cdot \mathrm{s}^{-1} \cdot \mathrm{Mpc}^{-1}$ one calculates $P=2.9 \times 10^{3} \mathrm{Mpc}=10.8 \times 10^{9}$ lightyears (ly).

Obviously, due to the four-dimensional deflection of the emitter from the three-dimensional tangential pseudo-Euclidean Minkowski space of the receiver, the mapping by light signals according to Peano must also be associated with an ambiguity of the latter mapping analogous to the Broglie formula on the quantum scale. Thereby, the cosmic redshift and the associated dimensionless apparent velocity $v_{\text {app }} / c$ according to Equation (42) in dependence on the magnitude of deflection from the receiver's tangential Minkowski space projection (and, therewith on distance) play the crucial role. From the preceding it is obvious that the Peano ambiguity of the cosmic redshift be referred to the latter's background rest frame (CMB).

Since in the closed $S^{3}$-hypersphere the redshift is observer-centric, exists for every observer a light horizon at distance $1 / 2 \pi P$, where $(1+z) \rightarrow \infty$, with geodesics $\Delta \sigma=1 / 4 \sigma=1 / 2 \pi P$ from the emitter to the receiver, both resting. According to ref. [15]: “... it is clear that the emitter's position, thus, must be deflected by value $P$ from the receiver's line of sight in tangentially projected Minkowski space, which is orthogonal to $P^{\prime}$. I.e., the bending of light-path through the warped space of the $S^{3}$-sphere causes the receiver to observe pseudo Euclidian projections (Minkowski space) of all ingoing geodesics $\Delta \sigma=1 / 2 \pi P$ in the direction of the tangent vector to the latter such that must be valid:

$$
\lambda_{P} \times \frac{\pi P}{2}=2 \pi P^{2} \lambda_{w} \times \frac{\pi P}{2}=\pi^{2} P^{3} \lambda_{w}
$$

I.e., we have a topological Peano mapping $\lambda_{P}$ from $\pi^{2} P^{3}$, the half of the hypersphere $2 \pi^{2} P^{3}$, with four-dimensional height $\lambda_{w}$ of the emitter via the four-dimensional geodesic $1 / 2 \pi P$ into the flat tangential Minkowski projection $2 \pi P^{2}$ with height $\lambda_{W}$ of the receiver, whereby the latter projection must be ambiguous since of the higher dimensionality of $\pi^{2} P^{3} \lambda_{w}$. Note that the farthest light is in-going at the receiver from distance $1 / 2 \pi P$ such that $(1+z) \rightarrow \infty$ and he, therewith, observes a two-dimensional semi-sphere on the plane of the sky. Hence, really applies:

$$
\lambda_{P}=\frac{2 \pi^{2} P^{3} \lambda_{w}}{\pi P}=2 \pi P^{2} \lambda_{w}
$$

Other than in quantum physics, the cosmic Peano ambiguity is not bound to the carrier of the mapping associated with a special physical situation on quantum scale, but only on the latter mapping $\lambda_{P}$ in the form of fluctuations of the cosmic redshift. One calculates $\lambda_{P}=36.0886 \times 10^{6}$ ly being according to Equation (58) equivalent to apparent recession velocity $V_{a p p}=819.96 \mathrm{~km} \cdot \mathrm{s}^{-1}$.

Clearly ambiguity waves with redshift amplitudes equivalent to the latter apparent recession velocity signify the Peano mapping of geodesics ingoing at the receiver from light horizons at distance $1 / 2 \pi P$ redshifted to $(1+z) \rightarrow \infty$. For distances smaller than $819.96 \mathrm{~km} \cdot \mathrm{s}^{-1}$ from the receiver in accord with Equation (58) are lower (decreasing) harmonics of the former ambiguity wave to expect in the order of

$$
\lambda_{\left(\frac{v_{a p p}}{c}\right)}<\lambda_{P}=\frac{\left(\frac{z_{P}}{2^{i} \ln 2}+1\right)^{2}-1}{\left(\frac{z_{P}}{2^{i} \ln 2}+1\right)^{2}+1}
$$

Reversely, for larger distances higher (increasing) harmonics should arise in the form of

$$
\lambda_{\left(\frac{v_{\text {app }}}{c}\right)}>\lambda_{P}=\frac{\left(z_{P} 2^{i} \ln 2+1\right)^{2}-1}{\left(z_{P} 2^{i} \ln 2+1\right)^{2}+1}
$$

where $i=1,2, \cdots, n$ and $z_{P}$ denotes redshift associated with apparent recession velocity $819.96 \mathrm{~km} \cdot \mathrm{s}^{-1} \mathrm{ac}-$ cording to Equation (58): 


$$
Z_{P}=\sqrt{\frac{\frac{819.96}{c}+1}{1-\frac{819.96}{c}}}-1=2.73884 \times 10^{-3}
$$

In the following calculations on the grounds of Equation (59) are compared with a survey of astronomical observations of redshift periodicities (e.g. Tifft, 1996 [30]):

$\begin{array}{lllllllll}\# \dot{i}: & 3 & 4 & 5 & 6 & 7 & 8 & 9 & 10 \\ \lambda_{P(\lambda)} \mathrm{km} \cdot \mathrm{s}^{-1}: & 148.01 & 74.08 & 37.04 & 18.52 & 9.26 & 4.63 & 2.32 & 1.16 \\ \text { Obs. } \lambda_{P(\lambda)}: & \approx 146.38 & \approx 73.19 & \approx 36.60 & \approx 18.30 & \approx 9.15 & \approx 4.57 & \approx 2.28 & -\end{array}$

For \#s $i=1$ and 2 correspondingly is calculated $590.53 \mathrm{~km} \cdot \mathrm{s}^{-1}$ and $296.48 \mathrm{~km} \cdot \mathrm{s}^{-1}$, respectively.

Quite obviously are most of the observationally derived redshift periodicities of Tifft and other researchers in accord with the preceding calculations according to Equation (59) (60).

According to Equation (60) basically also periodicities in the redshift of far-away galaxies and quasars should be observable, which indeed have been found by some researchers. The following synopsis compares predictions according to Equation (60) with some astrophysical observations [31-33]:

\begin{tabular}{llllllll} 
& \multicolumn{7}{c}{$\lambda_{\left(v_{\text {app }} / c\right)}>\lambda_{P}$} \\
$\# i:$ & 3 & 4 & 5 & 6 & 7 & 8 & 9 \\
$\lambda_{\left(v_{\text {app }} / c\right)}:$ & 0.0151 & 0.0299 & 0.0589 & 0.1142 & 0.2142 & 0.3766 & 0.5909 \\
$\lambda_{P(\text { i) }} z$ & 0.0152 & 0.0304 & 0.0607 & 0.1215 & 0.2431 & 0.4836 & 0.9720 \\
Obs. $Z$ : & - & $\approx 0.03$ & $\approx 0.06$ & $\approx 0.11$ & $\approx 0.258$ & $\approx 0.44$ & $\approx 1.0 / 1.1$
\end{tabular}

The also in the literature mentioned redshift periodicity at $z \approx 0.312$ e.g. can be explained by $z \approx 0.312$ $\approx 5 \times 0.0607 \approx 10 \times 0.0304 \approx 21 \times 0.0152$ etc.. But especially the observed accumulations of redshifts around $z \approx 0.63,1.2$ and 1.8 could be due to the accumulation of redshift periodicities at those values resulting from Equation (60). E.g. is calculated from the above $\lambda_{P(i)} Z$.

$$
\begin{gathered}
z \approx 0.63 \approx 42 \times 0.0152 \approx 21 \times 0.0304, \\
z \approx 1.2 \approx 5 \times 0.2431 \approx 10 \times 0.1215 \approx 20 \times 0.0607 \approx 40 \times 0.0304, \\
z \approx 1.8 \approx 1.9423 \approx 2 \times 0.9720 \approx 15 \times 0.1215 \approx 30 \times 0.0607,
\end{gathered}
$$

where $z=1.9423$ is referred to \# $i=10$ of Equation (60). Obviously, other reported periodicities can be explained analogously. For the succeeding \#s $i=11$ and 12 is calculated $z=3.8894$ and 7.7821, respectively. Note that especially the observed periodicities $\lambda_{\left(v_{\text {app }} / c\right)}>\lambda_{P}$ are not undisputed [34]. Anyhow, in the range $\lambda_{\left(v_{\text {app }} / c\right)}>\lambda_{P}$ the observational situation is much less definite than in the case $\lambda_{\left(v_{\text {app }} / c\right)}<\lambda_{P}$.

Eventually, it should be emphasized that the harmonics in the form of observer-centric redshift periodicities, according to the preceding, are not to understand as real accumulations of the respective observed cosmic objects, as e.g. galaxies or quasars, i.e., quantization of their distances. Rather, the former resonances represent Peano ambiguity of the redshift, implying ambiguity of the distances. This will say, that the distances derived from those redshifts are ambiguous too, i.e., they are defective. Furthermore, deliver the above equations a convincing reason for the anisotropic, undulating redshifts measured in the CMB (instead of the gravitational waves introduced in the early universe as part of the big bang model).

\section{CONCLUDING REMARKS}

The proposed unified, topological, realistic interpretation of all known wavelike patterns in optics, quantum physics and cosmic physics seems to picture the observationally ascertained physical phenomena 
in those physics domains quite well and generally confirms the especially in quantum physics developed mathematical tools. But this is not the case for the far reaching theoretical derivations from the latter formulae, referring to the nature of the wavelike behavior of matter and light. It is clear that the interpretation of Max Born in terms of density of probability is nearest to Peano's ambiguous mapping between manifolds of different dimensions, but without all other physical conclusions associated with the different, but equivalent mathematical models, especially developed by Heisenberg, Schrödinger, Feynman and Wigner. Physically real is only the Peano ambiguity, but not the mathematical model of the respective quantum physical situation.

Eventually should be remarked, that, analogously to the above final conclusion, in the case of micro physical mappings also by inversion of the topological argumentation, from the observer-centric quantization of the cosmic redshift, the proposed four-dimensional structure of the Universe conclusively follows as the most rational interpretation. Furthermore, from the preceding it is evident that the Peano ambiguity is valid only in all special natural and experimental physical situations on micro-physical scale, as well as in the case of the above discussed quantized cosmic redshift and associated dimensionless apparent velocity $V_{a p p} / \mathcal{c}$, where geometrically three-dimensional space-time physics comes in contact with the four-dimensionality of matter and the Universe, respectively. Therefore, a quantum theory on cosmological scale, i.e. a wave-function for the whole Universe, does not exist.

In summary, specifically for quantum physics, we also have shown the non-existence of:

- Bohr's principle of complementary;

- Schrödinger's real wave-packet with corpuscular properties;

- Heisenberg's dependence of corpuscular properties on the measurement process;

- Von Neumann's reduction of the hypothetical wave-function through conscious

Observation by a human being (intelligent creature);

- Everett's many-world hypothesis;

- De Broglie's and Bohm's hidden variables.

Rather it has been demonstrated that:

- Material Particles and photons are not mixed with any wave properties of which form ever;

- Waves in optics, quantum physics and in the light of very far cosmic objects naturally occur

Only as ambiguity of physical mappings between manifolds with difference of dimension

Equal to one;

- $\quad$ Those waves not really exist physically, but exclusively particles with and without rest mass

And certain properties geometrically and physically as well;

- All quantum mechanical systems always (at every time) have objective physical properties,

Independently of a measurement of the latter;

- The principle of causality is always maintained.

- A sudden spooky action over (considerable) distances in quantum-physics does not occur, so

Einstein is correct and Bohr and followers are wrong.

Finally: From the fact that the Peano ambiguity only occurs at the quantum physical level at the smallest possible distances, it also follows that this physical effect is of no importance for larger masses. Entanglement is not possible at this level.

\section{CONFLICTS OF INTEREST}

The author declares no conflicts of interest regarding the publication of this paper.

\section{REFERENCES}

1. De Broglie, L. (1925) Recherches sur la theorie des quanta (Researches on Quantum Theory). Annals de Physique, 3, 22. https://doi.org/10.1051/anphys/192510030022

2. Einstein, A. (1905) Über einen die Erzeugung und Verwandlung des Lichts betreffenden heuristischen Gesich- 
tspunkt (On a Heuristic Point of View Referring to the Generation and Change of Light). Annalen der Physik, 17, 132-148. https://doi.org/10.1002/andp.19053220607

3. Heisenberg, W. (1926) Über quantentheoretische Umdeutung kinematischer und mechanischer Beziehungen (On Quantum-Theoretical Reinterpretation of Kinematical and Mechanical Relations). Zeitschrift für Physik, 33, 897. https://doi.org/10.1007/BF01328377

4. Schrödinger, E. (1926) Quantisierung als Eigenwertproblem (Quantization as a Eigenvalue Problem). Annalen der Physik, 79, 361-376. https://doi.org/10.1002/andp.19263840404

5. Dirac, P.A.M. (1928) The Quantum Theory of the Electron. Proceedings of the Royal Society A, 117, 610. https://doi.org/10.1098/rspa.1928.0023

6. Born, M. (1926) Zur Quantenmecchanik der Stoßvorgänge (On Quantum Mechanics of Dispersion). Zeitschrift für Physik, 17, 863-867. https://doi.org/10.1007/BF01397477

7. Einstein, A., Podolsky, B. and Rosen, E. (1935) Can Quantum-Mechanical Description of Physical Reality Be Considered Complete? Physical Review, 47, 777-780. https://doi.org/10.1103/PhysRev.47.777

8. Bohr, N. (1935) Can Quantum-Mechanical Description of Physical Reality Be Considered Complete? Physical Review, 48, 696-702. https://doi.org/10.1103/PhysRev.48.696

9. Born, M. and Jordan, P. (1925) Zur Quantenmechanik (On Quantum-Mechanics). Zeitschrift für Physik, 34, 858. https://doi.org/10.1007/BF01328531

10. Bohm, D. (1952) A Suggested Interpretation of the Quantum Theory in Terms of Hidden Variables. Physical Review, 85, 166. https://doi.org/10.1103/PhysRev.85.166

11. Bell, J. (1966) On the Problem of Hidden Variables in Quantum Mechanics. Reviews of Modern Physics, 38, 447. https://doi.org/10.1103/RevModPhys.38.447

12. Fock, W. (1952) Kritik der Anschauungen Bohrs über die Quantenmechanik (Critique of Bohr's Views about Quantum Mechanics). Progress of Physics, 45, 3-14.

13. Everett, H. (1957) The Relative State Formulation of Quantum Mechanics. Reviews of Modern Physics, 29, 454-462. https://doi.org/10.1103/RevModPhys.29.454

14. Dirac, P.A.M. (1958) Principles of Quantum Mechanics. 4th Edition, Oxford University Press, Oxford.

15. Kunst, E.K. (2014) On the Physics inside a Closed, Static, Rotating Einsteinian Hypersphere in due Consideration of the Galaxy. Natural Science, 6, 897-961. https://doi.org/10.4236/ns.2014.611087

16. Lenz, F. (1951) The Ratio of Proton and Electron Masses. Physical Review, 82, 554. https://doi.org/10.1103/PhysRev.82.554.2

17. Van Wees, B.J., et al. (1988) Quantized Conductance of Point Contacts in a Two-Dimensional Electron Gas. Physical Review Letters B, 60, 848. https://doi.org/10.1103/PhysRevLett.60.848

18. Wharam, T.J., et al. (1988) One-Dimensional Transport and the Quantization of the Ballistic Resistance. Journal of Physics C, 21, L209. https://doi.org/10.1088/0022-3719/21/8/002

19. Thomas, K.J., et al. (1996) Interaction Effects in a One-Dimensional Constriction. Physical Review Letters, 77, 135. https://doi.org/10.1103/PhysRevLett.77.135

20. von Klitzing, K., et al. (1980) New Method for High Accuracy Determination of the Fine Structure Constant Based on Quantized Hall Resistance. Physical Review Letters, 45, 494-497. https://doi.org/10.1103/PhysRevLett.45.494

21. Störmer, H.L. and Hill, M. (1984) Der fraktionale QHE (The Fractional QHE). Phys. Blätter, Nr. 9.

22. Novoselov, H.L., et al. (2005) Two-Dimensional Gas of Massless Dirac Fermions in Graphene. Nature, 438, 197-200. https://doi.org/10.1038/nature04233 
23. Cantor, G. (1878) Ein Beitrag zur Mannigfaltigkeitslehre (A Contribution to the Theory of Manifoldness). Journal für die reine und angewandte Mathematik, 84, 242-258. https://doi.org/10.1515/crll.1878.84.242

24. Peano, G. (1890) Sur une courbe qui remplit toute une aire plane (On a Curve That Fills a Whole Flat Surface). Mathematische Annalen, 36, 157-160. https://doi.org/10.1007/BF01199438

25. Brouwer, L.E.J. (1911) Beweis der Invarianz der Dimensionszahl (Proof of Invariance of Dimension). Mathematische Annalen, 70, 161-165. https://doi.org/10.1007/BF01461154

26. Bach, R., et al. (2013) Controlled Double-Slit Electron Diffraction. New Journal of Physics, 15, Article ID: 033018. http://www.njp.org https://doi.org/10.1088/1367-2630/15/3/033018

27. Fuwa, M., et al. (2015) Experimental Proof of Nonlocal Wavefunction Collapse for a Single Particle Using Homodyne Measurements. Nature Communication, 6, Article No. 66655. https://doi.org/10.1038/ncomms7665

28. Robens, C., et al. (2015) Ideal Negative Measurements in Quantum Walks Disprove Theories Based on Classical Trajectories. Physical Review X, 5, Article ID: 0110032015. https://doi.org/10.1103/PhysRevX.5.011003

29. Kunst, E.K. (2021) Relativistic Quantitative Determination of the "Mysterious" Differences in the Hubble Constant. Natural Science, 13, 1-7. https://doi.org/10.4236/ns.2021.131001

30. Tifft, W.G. (1996) Evidence for Quantized and Variable Redshifts in the Cosmic Background Rest Frame. Astrophysics and Space Science, 244, 29-56. https://doi.org/10.1007/BF00642278

31. Karlsson, K.G. (1971) Possible Discretization of Quasar Redshifts. Astronomy \& Astrophysics, 13, 333-335.

32. Bell, M.B. and Mc Diarmid, D. (2006) Six Peaks Visible in the Redshift Distribution of 46,400 Sdss Quasars Agree with the Preferred Redshifts Predicted by the Decreasing Intrinsic Redshift Model. The Astrophysical Journal, 648, 140. https://doi.org/10.1086/503792

33. Hartnett, J.G. (2008) Redshift Periodicity in Quasar Number Counts from Sloan Digital Sky Survey.

34. Tang, S.M. and Zhang, S.N. (2005) Critical Examinations of QSO Redshifts Periodicities and Associations with Galaxies in Sloan Digital Sky Survey Data. The Astrophysical Journal, 633, 41-51.

https://doi.org/10.1086/432754 